\title{
Poly (ADP-Ribose) Polymerase Mediates Diabetes-Induced Retinal Neuropathy
}

\author{
Ghulam Mohammad, Mohammad Mairaj Siddiquei, and Ahmed M. Abu El-Asrar \\ Department of Ophthalmology, College of Medicine, King Saud University, P.O. Box 245, Riyadh 11411, Saudi Arabia \\ Correspondence should be addressed to Ghulam Mohammad; gmkbiochembhu@gmail.com
}

Received 4 August 2013; Revised 30 October 2013; Accepted 3 November 2013

Academic Editor: Dorota Raczynska

Copyright ( 2013 Ghulam Mohammad et al. This is an open access article distributed under the Creative Commons Attribution License, which permits unrestricted use, distribution, and reproduction in any medium, provided the original work is properly cited.

\begin{abstract}
Retinal neuropathy is an early event in the development of diabetic retinopathy. One of the potential enzymes that are activated by oxidative stress in the diabetic retina is poly (ADP-ribose) polymerase (PARP). We investigated the effect of the PARP inhibitor 1,5-isoquinolinediol on the expression of the neurodegeneration mediators and markers in the retinas of diabetic rats. After two weeks of streptozotocin-induced diabetes, rats were treated with 1,5-isoquinolinediol ( $3 \mathrm{mg} / \mathrm{kg} / \mathrm{day})$. After 4 weeks of diabetes, the retinas were harvested and the levels of reactive oxygen species (ROS) were determined fluorometrically and the expressions of PARP, phosporylated-ERK ${ }_{1 / 2}$, BDNF, synaptophysin, glutamine synthetase (GS), and caspase-3 were determined by Western blot analysis. Retinal levels of ROS, PARP-1/2, phosphorylated $\mathrm{ERK}_{1 / 2}$, and cleaved caspase-3 were significantly increased, whereas the expressions of BDNF synaptophysin and GS were significantly decreased in the retinas of diabetic rats, compared to nondiabetic rats. Administration of 1,5-isoquinolinediol did not affect the metabolic status of the diabetic rats, but it significantly attenuated diabetesinduced upregulation of PARP, ROS, ERK I/2 $_{2}$ phosphorylation, and cleaved caspase- 3 and downregulation of BDNF, synaptophysin, and GS. These findings suggest a beneficial effect of the PARP inhibitor in increasing neurotrophic support and ameliorating early retinal neuropathy induced by diabetes.
\end{abstract}

\section{Introduction}

Diabetes is a metabolic disorder characterized by hyperglycemia and often leads to numerous microvascular complications, including retinopathy. Diabetic retinopathy (DR) is a multifactorial disease, and persistent hyperglycemia appears to be a major contributor to its development. Recent evidence suggests that diabetic retinopathy is a progressive neurodegenerative disease as evidenced by the presence of apoptotic cells in all retinal layers. Activation of caspase-3 is part of the mechanism of apoptosis. Visual dysfunction is initiated early after the onset of diabetes and progresses independently of the vascular lesions [1-4]. The exact molecular mechanisms which contribute to development of diabetes-induced retinal neuropathy remain largely unknown. Reactive oxygen species (ROS) production is increased in the retina in diabetes, and it is considered as one of the major contributors of retinal metabolic abnormalities postulated to be involved in the development of diabetic retinopathy. Administration of antioxidants to diabetic rats protects the retina from oxidative damage, and also the development of retinopathy [5-8]. In diabetes, retinal neuropathy is associated with enhanced oxidative stress resulting from excess generation of ROS that often leads to retinal microvascular cell death $[3,9,10]$.

Enhanced ROS level causes reduced levels of brainderived neurotrophic factor (BDNF), a protein belonging to the neurotrophin family. BDNF is expressed in retinal ganglion cells (RGCs) and Müller cells [11] and is important for the survival of retinal ganglion cells [12]. BDNF is important for neural development and cell survival and is essential for molecular mechanisms of synaptic activity [13]. Recent studies suggested that the early retinal neuropathy of diabetes involves the reduced expression of BDNF and can be ameliorated by an exogenous supply of this neurotrophin [1, 3]. ROS also decreases the level of synaptophysin, a synaptic vesicle protein for neurotransmitter release which is widely 
expressed in the retina $[14,15]$. Glutamate, the excitatory neurotransmitter in the retina, is released by photoreceptors, bipolar cells, and ganglion cells and mediates the transfer of visual signals from the retina to the brain [16]. Excess glutamate release in hypoxic-ischemic conditions causes excitotoxic damage to the RGCs through activation of ionotropic and metabotropic glutamate receptors. The synaptically released glutamate is taken up by Müller cells where glutamine synthetase converts it into glutamine. Several studies found that the expression of GS was significantly decreased in the diabetic rat retinas $[17,18]$. These dysfunctions resulted in elevated glutamate levels in the diabetic retinas $[17,19,20]$, which might induce retinal neurodegeneration via glutamate excitotoxicity. Synaptophysin protein is decreased in the retina of the streptozotocin (STZ)-induced diabetes model through the ROS-extracellular signal-regulated kinase 1 and $2\left(\mathrm{ERK}_{1 / 2}\right)$ suggesting the involvement of cross talk between mitogen-activated protein kinases (MAPK) pathway signals and neurodegeneration in the diabetic retina $[3,21]$. It was also demonstrated that the reduction of BDNF and synaptophysin in the diabetic retina was attenuated by the antioxidant lutein, indicating that this change was partly caused by excessive oxidative stress [3]. One of the major consequences of oxidative stress is DNA damage. High level of ROS induces DNA strand breaks in the retina by hyperglycemia [22], and ROS-induced DNA single-strand breakages were considered an obligatory step for Poly(ADP-ribose) polymerase (PARP) cleavage/activation.

PARP is a nuclear enzyme that regulates several cellular events including DNA repair, cellular division and differentiation, DNA replication, transformation, gene expression and amplification, mitochondrial function, and cell death. Altered activity of PARP is reported under many pathological conditions, including diabetes. Extensive experimental data generated in both tissue culture and animal models signify that diabetes-induced PARP activation or its overexpression in the retina by DNA damage induces cell death; a phenomenon that precedes the development of histopathologic change [22-25]. Recently, it was documented that PARP activation contributes to superoxide anion radical and peroxynitrite formation in peripheral nerve, vasa nervorum, and aorta of STZ-induced diabetic rats and high-glucose exposed human Schwann cells [26]. Furthermore, PARP inhibition counteracted diabetes-induced systemic oxidative stress and 4-hydroxynonenal adduct accumulation in peripheral nerves and improved nerve fiber function [27]. These findings suggest that PARP activation in diabetes is bi- rather than unidirectional. However, so far no study has shown the cross talk between PARP activation and retinal neuropathy. Therefore, in this study, we explored the hypothesis that PARP activation mediates retinal neuropathy by reducing BDNF, synaptophysin, and GS levels in the diabetic retina. To test this hypothesis, we measured the levels of ROS generation, PARP, phosphorylated $\mathrm{ERK}_{1 / 2}\left(\mathrm{p}-\mathrm{ERK}_{1 / 2}\right), \mathrm{BDNF}$, synaptophysin, GS, and cleaved caspase-3 in the retina of diabetic animals. In addition, we analyzed whether treatment with the PARP inhibitor 1,5-isoquinolinediol suppresses the neurodegenerative changes in the retinas of diabetic rats.

\section{Materials and Methods}

2.1. Induction of Diabetes and 1,5-Isoquinolinediol Treatment. All procedures with animals were performed in accordance with the Association for Research in Vision and Ophthalmology (ARVO) statement for use of animals in ophthalmic and vision research and were approved by the institutional animal care and use committee of the College of Pharmacy, King Saud University. Adult male Sprague Dawley rats of 89 weeks of age (200-220 g) were overnight fasted and STZ $65 \mathrm{mg} / \mathrm{kg}$ in $10 \mathrm{mM}$ sodium citrate buffer, pH 4.5 (Sigma, St. Louis, MO, USA) was injected intraperitoneal. Equal volumes of citrate buffer were injected in nondiabetic animals. Rats were considered diabetic if their blood glucose was greater than $250 \mathrm{mg} / \mathrm{dL}$. Age-matched normal rats served as controls.

Two weeks later, the diabetic rats were randomly divided into 2 groups matched for body weight and blood glucose: diabetic group without treatment $(\mathrm{D}, \mathrm{N}=10)$ and diabetic group given 1,5-isoquinolinediol $(3 \mathrm{mg} / \mathrm{kg} /$ day, intraperitoneal; Santa Cruz Biotechnology, CA) (Dib + ISO, N = 10). This dose of 1,5-isoquinolinediol was based on previous studies [28]. After 4 weeks of diabetes, the rats were euthanized by an overdose of chloral hydrate, the eyes were removed, and retina was isolated and frozen immediately in liquid nitrogen and stored at $-80^{\circ} \mathrm{C}$ to be analyzed by Western blot analysis or biochemical assay.

2.2. Immunohistochemical Analysis. The C57Bl/6J mice were made diabetic by intraperitoneal injection of Streptozotocin for five consecutive days. Mice with blood glucose above $200 \mathrm{mg} / \mathrm{dL}, 3$ days after the last injection of streptozotocin, were considered as diabetic. Age-matched normal C57Bl/6J mice served as controls. After 12 weeks of diabetes, the mice were euthanized by an overdose of chloral hydrate, the eyes were removed, and were used for immunohistochemistry by incubation in $10 \%$ paraformaldehyde for $30 \mathrm{~min}$. Then the eyes were washed with PBS, fixed in optimal cutting temperature compound (OCT), and immediately frozen in liquid nitrogen for sectioning. Cryosections $(10 \mu \mathrm{m})$ prepared from mouse retina were fixed with $4 \%$ paraformaldehyde and blocked with $10 \%$ normal goat serum and incubated with rabbit polyclonal anti-PARP antibody overnight (1:150, Cat. no. SC-7150, Santa Cruz Biotechnology, Inc., Santa Cruz, CA, USA). After rinsing the slides with PBS, these were incubated with the secondary antibody (anti-rabbit-FITC conjugated) for 1 hour. The slides were rinsed with PBS, mounted with DAPI-containing mounting media (Vector Laboratories), and imaged with an Olympus BX-UCB fluorescent microscope (20x magnification).

2.3. Western Blot Analysis. Retinas were homogenized in a western lysis buffer $(30 \mathrm{mM}$ Tris- $\mathrm{HCl}, \mathrm{pH} 7.4,250 \mathrm{mM}$ $\mathrm{Na}_{3} \mathrm{VO}_{4}, 5 \mathrm{mM}$ EDTA, $250 \mathrm{mM}$ sucrose, $1 \%$ Triton X100 with Protease inhibitor). The lysate was centrifuged at $14,000 \times \mathrm{g}$ for $10 \mathrm{~min}$ at $4^{\circ} \mathrm{C}$, and the supernatant was collected. Protein content was assayed by DC protein assay (BioRad Laboratories, Hercules, CA, USA). The tissue lysates containing $40-50 \mu \mathrm{g}$ protein were separated on $8-15 \%$ SDSpolyacrylamide gels and were transferred onto nitrocellulose 
membranes. The blots were blocked with $5 \%$ nonfat milk in TBST (20 mM Tris- $\mathrm{HCl}, \mathrm{pH}$ 7.6, $136 \mathrm{mM} \mathrm{NaCl}$, and $0.1 \%$ Tween-20).

For detection of PARP, p-ERK $1 / 2$, BDNF, synaptophysin, and cleaved caspase- 3 , the membrane was incubated overnight at $4^{\circ} \mathrm{C}$ with mouse monoclonal anti-PARP $(1: 200$, Cat. no. ab110915, Abcam, UK), rabbit polyclonal anti-ERK $1 / 2$ (1:300, Cat. no. ab17942, Abcam), mouse monoclonal antiphosphorylated $\mathrm{ERK}_{1 / 2}(1: 300$, Cat. no. ab50011, Abcam), mouse monoclonal anti-BDNF (1:500, Cat. no. SC-65513, Santa Cruz Biotechnology), goat polyclonal anti-synaptophysin $(1 \mu \mathrm{g} / \mathrm{mL}$, Cat. no. AF-5555, R\&D Systems, Minneapolis, MN, USA), goat polyclonal anti-GS (1:500, SC-6640, Santa Cruz Biotechnology), and rabbit monoclonal anticaspase-3 (1:300, Cat. no. MAB835, R\&D Systems). After overnight incubation with primary antibodies, the membranes were washed four times with TBS-T ( 5 min each). For PARP, p-ERK ${ }_{1 / 2}$, and BDNF, the membrane was incubated at room temperature for $1.5 \mathrm{~h}$ with anti-mouse secondary horseradish peroxidase-conjugated antibody $(1: 2000$, SC2005, Santa Cruz Biotechnology), for synaptophysin with anti-goat secondary horseradish peroxidase-conjugated antibody (1:2000, SC-2768, Santa Cruz Biotechnology), and for $\mathrm{ERK}_{1 / 2}$ and cleaved caspase-3, with anti-rabbit secondary horseradish peroxidase-conjugated antibody $(1: 2000$, SC2004, Santa Cruz Biotechnology). After incubations with secondary antibodies, membranes were washed four times with TBS-T (5 min each) and the immunoreactivity of bands was visualized on a high-performance chemiluminescence machine (G: Box Chemi-XX8 from Syngene, Synoptic Ltd. Cambridge, UK) by using enhanced chemiluminescence plus Luminol (SC-2048, Santa Cruz Biotechnology) and quantified by densitometric analysis using image processing and analysis in GeneTools (Syngene by Synoptic Ltd. Cambridge, UK). As a control, the blots were stripped and detected with a mouse monoclonal anti- $\beta$-actin $(1: 2000$, SC-47778, Santa Cruz Biotechnology), antibody. All data from the three independent experiments were expressed as a ratio to OD.

\subsection{Reactive Oxygen Species Measurements. Reactive Oxy-} gen Species (ROS) generation was measured in retinal tissue homogenates using a $2^{\prime}, 7^{\prime}$-dichlorofluorescein-diacetate (DCHFDA) [29]. DCFHDA, a nonfluorescent dye, is cleaved by esterase activity to yield DCFH, which is subsequently oxidized by a variety of ROS to form dichlorofluorescein (DCF), which is fluorescent. Retinas were homogenized in PBS in presence of protease inhibitor using a glass homogenizer. Samples containing $20 \mu \mathrm{g}$ proteins diluted in PBS were incubated with $5 \mu \mathrm{M}$ DCFHDA (Invitrogen, CA, USA) in the dark for $15 \mathrm{~min}$. Fluorescence was measured using a spectraMax Gemini-XPS (Molecular Devices, CA, USA) every $15 \mathrm{~min}$ for $1 \mathrm{~h}$ with excitation and emission wavelengths of $488 \mathrm{~nm}$ and $525 \mathrm{~nm}$.

2.5. Apocynin Treatment. STZ-induced diabetic rats were divided into 2 groups: the rats in group I received normal drinking water without any supplementation, and those in group II received drinking water supplemented with apocynin $(15 \mathrm{mg} / \mathrm{kg} /$ day in drinking water; Santa Cruz Biotechnology) immediately after establishment of diabetes. This dose of apocynin was based on previous studies [30]. Each group had 8-12 rats. After 4 weeks of diabetes, the rats were euthanized by an overdose of chloral hydrate, the eyes were removed, and retina was isolated and frozen immediately in liquid nitrogen and stored at $-80^{\circ} \mathrm{C}$ to be analyzed by Western blot analysis or biochemical assay.

2.6. Statistical Analysis. Each measurement was made in duplicate, and the assay was repeated three or more times. Data are expressed as mean \pm SD and experimental groups were compared using the nonparametric Kruskal-Wallis test followed by the Mann-Whitney test for multiple-group comparison. A $P$ value $\leq 0.05$ indicated statistical significance. SPSS version 12.0 was used for the statistical analyses.

\section{Results}

3.1. Metabolic Changes Induced by Diabetes. The body weights of the diabetic rats were significantly lower and their blood glucose values were more than fourfold higher compared with age-matched normal control rats $(170 \pm 22$ versus $270 \pm 28 \mathrm{~g}$ and $453 \pm 32$ versus $111 \pm 12 \mathrm{mg} / \mathrm{dL}$, resp.). 1,5-isoquinolinediol treatments to diabetic rats for 2 weeks did not affect the body weight and blood glucose levels compared to untreated diabetic rats $(182 \pm 25$ versus $170 \pm$ $22 \mathrm{~g}$ and $469 \pm 36$ versus $453 \pm 32 \mathrm{mg} / \mathrm{dL}$ ).

3.2. Effect of Diabetes on Retinal Expression and Activation of PARP. Retinal PARP immunoreactivity (green FITC fluorescent staining) was upregulated in diabetic rats compared with nondiabetic controls. PARP was localized primarily in the ganglion cell and inner nuclear layers of the retina (Figure 1(a)). Representative Western blot image of retinal poly(ADP-ribosyl)ated proteins in control and diabetic rats was maintained with and without PARP inhibitor treatment (Figure 1(b)). Western blot analysis demonstrated significant upregulation of PARP-1/2 expression in diabetic retinas compared to nondiabetic retinas. The expression of PARP$1 / 2$ protein in the retinas of diabetic rats was upregulated by about $40 \%$ as compared to the retinas of nondiabetic rats (Figure 1(c)).

3.3. Effect of Diabetes on Retinal Expression of Mediators and Markers of Neurodegeneration. Western blot analysis demonstrated significant upregulation of $\mathrm{ERK}_{1 / 2}$ phosphorylation in diabetic retinas compared to nondiabetic retinas. The phosphorylation of $\mathrm{ERK}_{1 / 2}$ protein in the retinas of diabetic rats was upregulated by about $45 \%$ as compared to the retinas of nondiabetic rats (Figure 2). The neurotrophin BDNF was significantly downregulated in diabetic retinas compared to nondiabetic controls. BDNF expression in the retinas of diabetic rats was decreased by about $52 \%$ compared to nondiabetic controls (Figure 3). The expression of the synaptic vesicle protein synaptophysin in the retinas of diabetic rats was downregulated by about $35 \%$ as compared to the retinas of nondiabetic rats (Figure 4). GS is an enzyme 

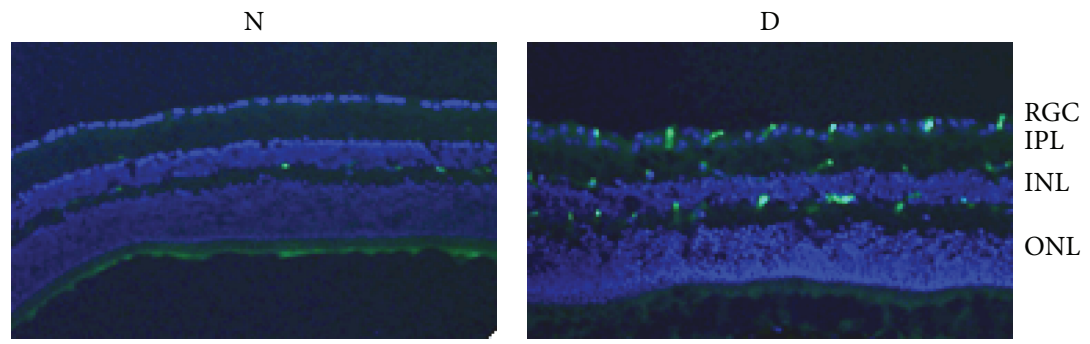

(a)

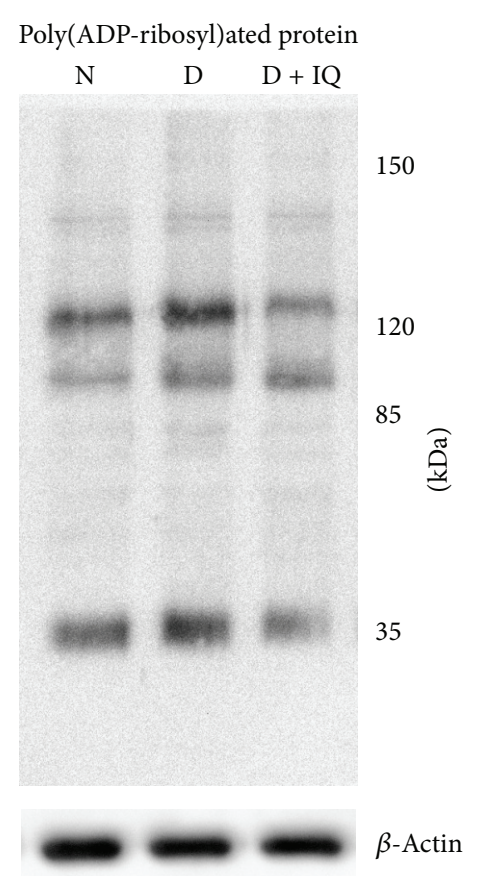

(b)
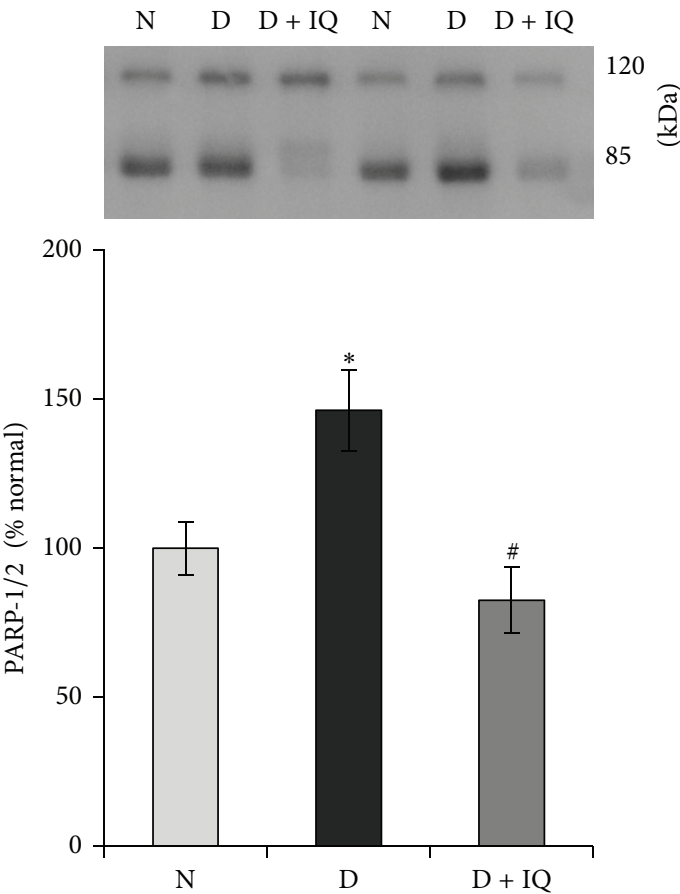

(c)

FIGURE 1: Effect of 1,5-isoquinolinediol on retinal poly(ADP-ribose) polymerase (PARP) expression in diabetes. (a) Cryosections from normal $(\mathrm{N})$ and diabetic (D) retina were subjected to immunostaining using anti-PARP antibodies (green) and DAPI (blue) was used to stain the nuclei. The sections were imaged at 20x magnification using Olympus BX-UCB fluorescent microscope. RGC: retinal ganglion cell; IPL: inner plexiform layer; INL: inner nuclear layer; ONL: outer nuclear layer. (b) Representative Western blot analyses of retinal poly(ADP-ribosyl)ated proteins in control and diabetic rats maintained with and without PARP inhibitor treatment. (c) PARP activation was measured by western blot technique, and the ratio of active PARP-2 $(85 \mathrm{KDa})$ and pro-PARP-1 $(120 \mathrm{kDa})$ was calculated in control and diabetic rats maintained with and without PARP inhibitor treatment. Measurements were made in duplicate in six to eight rats in each group. Western blots are representative of three different experiments. Results are expressed as mean \pm SD. Values obtained from nondiabetic rats are considered as $100 \%$. N: nondiabetic rat; D: diabetic rat; D + IQ: diabetic rat treated with 1,5-isoquinolinediol. ${ }^{*} P<0.05$ compared with nondiabetic rat. ${ }^{\#} P<0.05$ compared with diabetic rat.

which converts glutamate into glutamine; protein expression was significantly decreased by about $58 \%$ in diabetic retinas compared to nondiabetic retinas (Figure 5). Cleaved caspase3 , the apoptosis executer enzyme, was significantly upregulated in diabetic retinas compared to nondiabetic controls. Cleaved caspase-3 levels in the retinas of diabetic rats were increased by about $65 \%$ compared to nondiabetic controls (Figure 6).

3.4. Effect of Diabetes on Retinal ROS Generation. Spectrofluorometric analysis demonstrated significant upregulation of ROS generation in diabetic retinas compared to nondiabetic retinas. ROS production in retinas was determined by
DCFH-DA probe. The levels of DCF fluorescence intensity in the retina of diabetic rats were increased by $40 \%$ as compared to control rats (Figure 7).

\subsection{The PARP Inhibitor 1,5-Isoquinolinediol Attenuates} Diabetes-Induced PARP Upregulation. 1,5-isoquinolinediol is a potent PARP antagonist and decreases PARP activity in diabetic retina [28]. We employed 1,5-isoquinolinediol to investigate the antineurodegeneration function in the retinas of diabetic rats. Diabetic rats that were treated with 1,5-isoquinolinediol showed significant attenuation of PARP- $1 / 2$ activation by about $60 \%$ as compared to untreated diabetic rats (Figure 1(c)). 


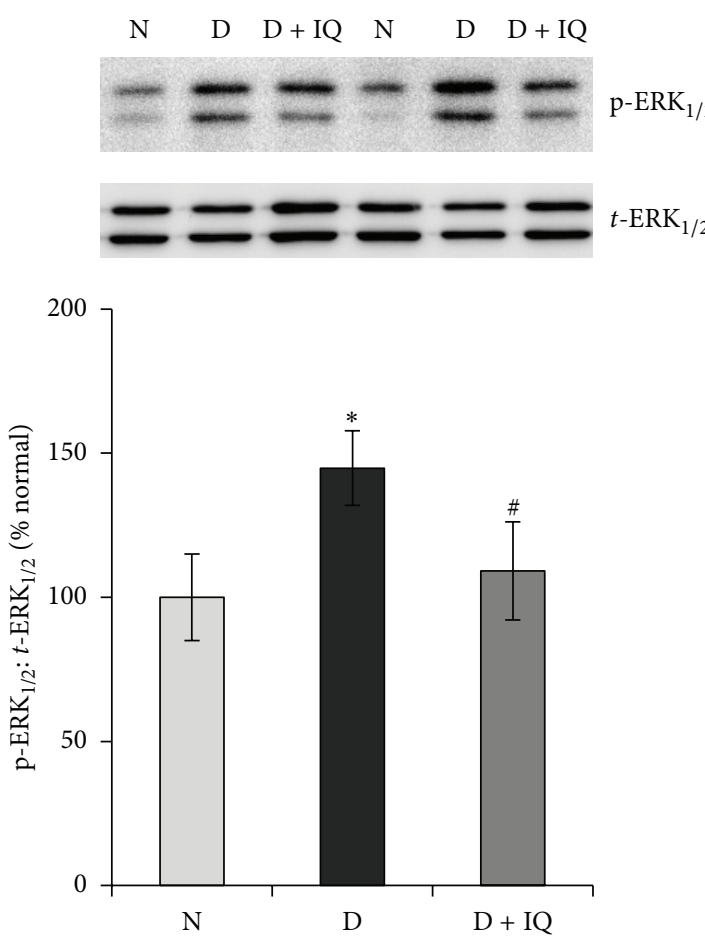

FIGURE 2: Effect of 1,5-isoquinolinediol on retinal ERK $\mathrm{E}_{1 / 2}$ activation in diabetes. Relative abundance of phosphorylated $\mathrm{ERK}_{1 / 2}$ (p$\left.\mathrm{ERK}_{1 / 2}\right)$ and total $\mathrm{ERK}_{1 / 2}\left(t-\mathrm{ERK}_{1 / 2}\right)$ was determined by Western blotting, followed by densitometry. Data are expressed as percentage change in phosphorylation over $t$-ERK $\mathrm{ER}_{1 / 2}$ and are expressed as mean \pm SD. Values obtained from nondiabetic rats are considered as $100 \%$. $\mathrm{N}$ : nondiabetic rat; $\mathrm{D}$ : diabetic rat; $\mathrm{D}+\mathrm{IQ}$ : diabetic rat treated with 1,5 -isoquinolinediol. ${ }^{*} P<0.05$ compared with nondiabetic rat. ${ }^{\#} P<0.05$ compared with diabetic rat.

3.6. The PARP Inhibitor 1,5-Isoquinolinediol Attenuates the Effect of Diabetes. Western blot analysis was used to assess the effect of 1,5-isoquinolinediol on diabetes-induced alterations of $\mathrm{p}-\mathrm{ERK}_{1 / 2}, \mathrm{BDNF}$, synaptophysin, GS, cleaved caspase-3, and ROS. 1,5-isoquinolinediol administration significantly attenuated diabetes-induced upregulation of $\mathrm{p}-\mathrm{ERK}_{1 / 2}$ and cleaved caspase- 3 by about $40 \%$ and $60 \%$, respectively. In the 1,5-isoquinolinediol-treated diabetic rats, the decrease in BDNF, synaptophysin, and GS caused by diabetes was attenuated by about $50 \%, 34 \%$, and $50 \%$, respectively (Figures $2-6$ ). The levels of ROS generation are attenuated by about $35 \%$ in the retina of diabetic rats treated by 1,5 -isoquinolinediol as compared to untreated diabetic rats (Figure 7).

3.7. Effect of the Specific ROS Inhibitor Apocynin on PARP1/2 Expression. Several previous studies have shown that PARP activation is mediated via excess ROS generation [31]. Therefore, we determined the expression levels PARP$1 / 2$ expression in retina from the control, diabetic, and apocynin-treated rats. Pooled data accrued from multiple retinal preparations, as determined by Western blotting and densitometry, indicated a significant upregulation in the expression of PARP-1 in the diabetic retina compared with the control. Constant apocynin intake from the onset of diabetes

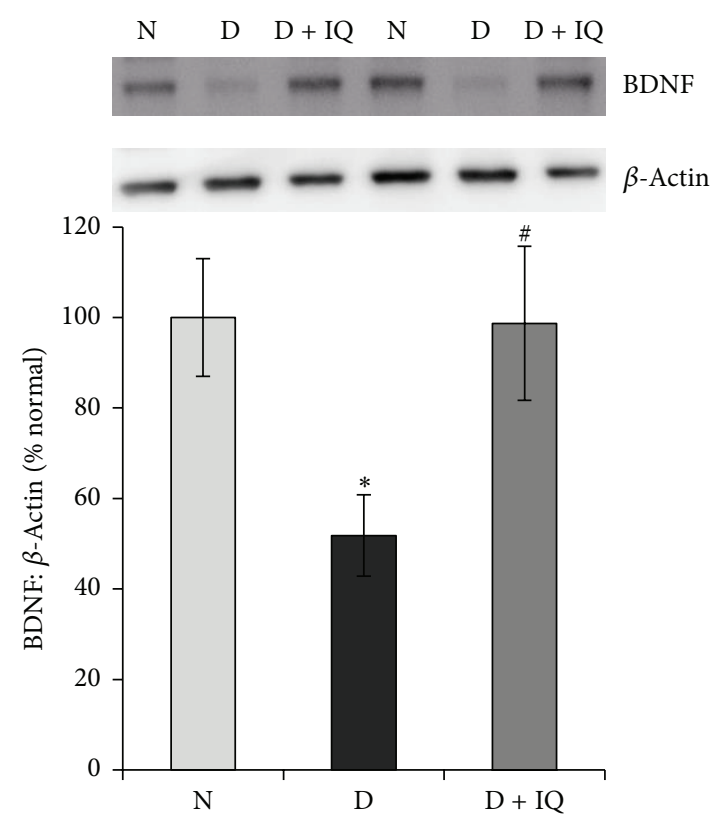

FIGURE 3: Effect of 1,5-isoquinolinediol on retinal brain-derived neurotrophic factor (BDNF) expression in diabetes. The expression of BDNF in retinal homogenate was determined by Western blotting technique. The histogram represents the mean band intensity (from $6-8$ rats in each group) of BDNF adjusted to the intensity of $\beta$ actin in the same sample. Values obtained from nondiabetic rats are considered as $100 \%$. N: nondiabetic rat; D: diabetic rat; D + IQ: diabetic rat treated with 1,5 -isoquinolinediol. ${ }^{*} P<0.05$ compared with nondiabetic rat. ${ }^{\#} P<0.05$ compared with diabetic rat.

significantly attenuated diabetes-induced upregulation of PARP-1/2 (Figure 8).

\section{Discussion}

In the present study, we investigated the role of PARP inhibition on diabetes-induced retinal neuropathy. We demonstrated that in the diabetic retina, PARP activation, ROS, p-ERK $K_{1 / 2}$, and cleaved caspase-3 levels were significantly increased. On the other hand, diabetes induced significant downregulation of the neurotrophin BDNF, the synaptic function marker synaptophysin, and GS. Administration of PARP inhibitor to diabetic rats significantly attenuated diabetes-induced increased expressions of PARP activation, ROS, p-ERK $K_{1 / 2}$, and cleaved caspase- 3 and decreased BDNF, synaptophysin, and GS levels in the retinas.

Here, we report that retinal PARP immunoreactivity was upregulated in diabetic rats compared with nondiabetic controls. PARP immunoreactivity was localized primarily in the ganglion cell and inner nuclear layers of the retina. Similarly, the protein expressions of PARP-1/2 were upregulated by diabetes in the retina. Our results are consistent with previous reports that demonstrated upregulation of PARP expression in the diabetic retinas [28]. Here, we also report that diabetes-induced upregulation of PARP expression in the retina was prevented by the specific ROS inhibitor apocynin. Our results are also in agreement with previous reports that 


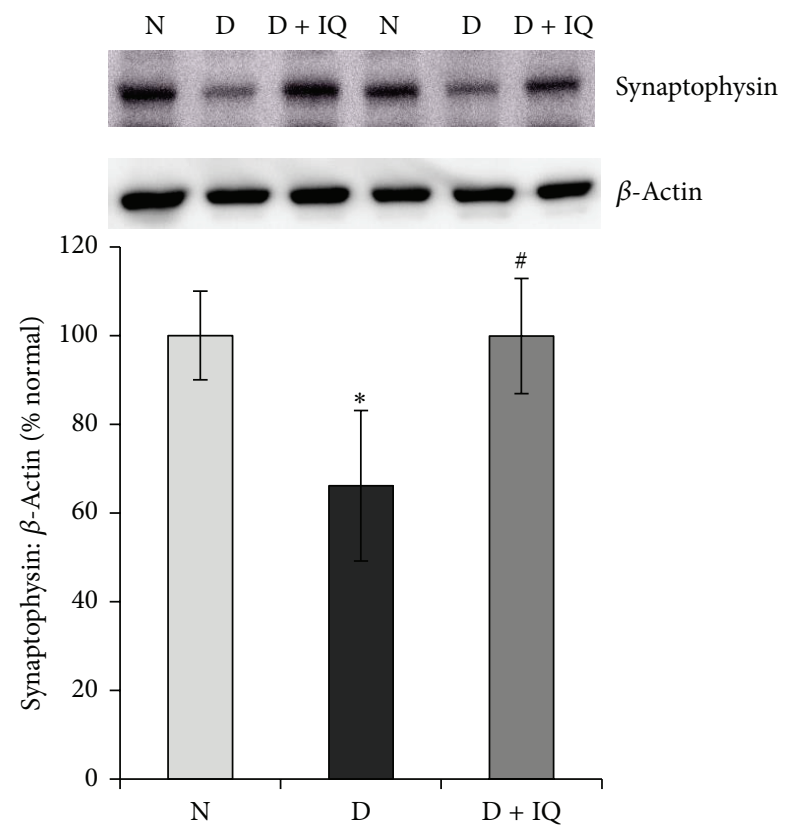

FIGURE 4: Effect of 1,5-isoquinolinediol on retinal synaptophysin expression in diabetes. The expression of synaptophysin in retinal homogenates was determined by Western blotting technique. The histogram represents the mean band intensity (from 6-8 rats in each group) of synaptophysin adjusted to the intensity of $\beta$-actin in the same sample. Values obtained from nondiabetic rats are considered as $100 \%$. N: nondiabetic rat; D: diabetic rat; D + IQ: diabetic rat treated with 1,5 -isoquinolinediol. ${ }^{*} P<0.05$ compared with nondiabetic rat. ${ }^{\#} P<0.05$ compared with diabetic rat.

demonstrated increased levels of ROS and PARP in STZinduced diabetic rat retinas [22, 23, 32]. Zheng et al. [33] reported that excessive oxidative stress is responsible for the upregulation of PARP in the diabetic retinas. Furthermore, supplementation of antioxidants such as simvastatin significantly attenuated diabetes-induced ROS generation, PARP activation, retinal capillary cells apoptosis, and formation of acellular capillaries in the retina. It was also shown that the early retinal changes induced by diabetes involve the overexpression of PARP, whose activation is associated with a retinal capillary cell apoptosis [22].

We showed here that the ROS level was reduced in the retinas of diabetic rats treated with the PARP inhibitor. Thus, our findings suggest that diabetes-induced ROS production in the retina appears to be also mediated by PARP. Our results are in agreement with previous reports that demonstrated that PARP inhibition attenuates the erectile impairment in diabetic rats by inhibition of ROS generation [34]. Similarly, Szabó et al. [35] suggested that ROS generation is a downstream target of hyperglycemia-induced PARP activation, as PARP inhibitors blocked the hyperglycemia-induced ROS generation in podocytes. Taken together, these findings suggest the presence of a positive feedback regulation between PARP activation and ROS generation in the diabetic retina.

The principal mediator responsible for the dysregulation of neurodegenerative markers such as BDNF, synaptophysin,

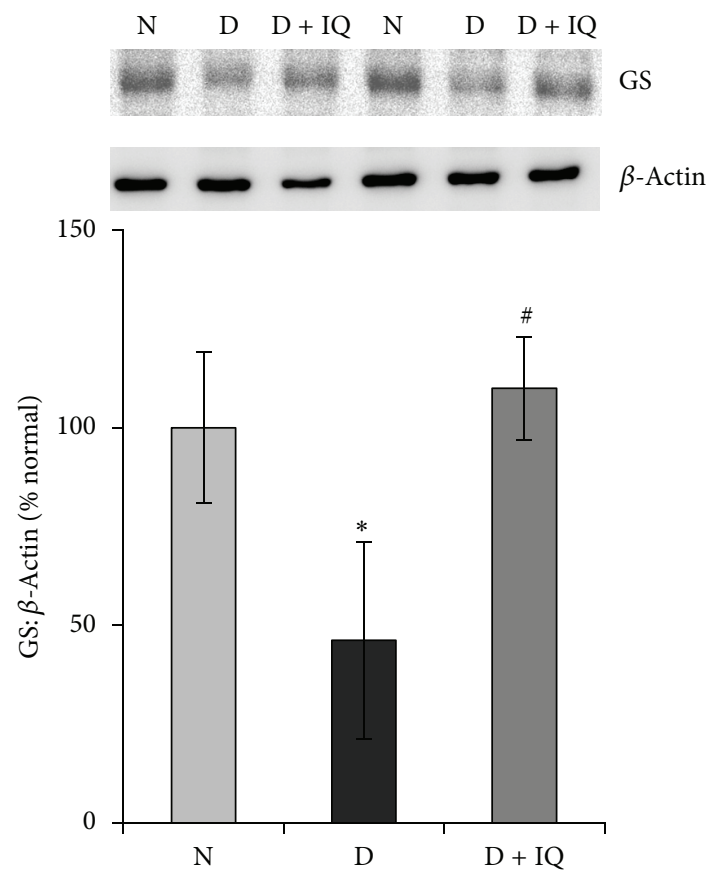

FIGURE 5: Effect of 1,5-isoquinolinediol on retinal GS expression in diabetes. The expression of GS in retinal homogenates was determined by Western blotting technique. The histogram represents the mean band intensity (from 6-8 rats in each group) of GS adjusted to the intensity of $\beta$-actin in the same sample. Values obtained from nondiabetic rats are considered as $100 \%$. N : nondiabetic rat; D: diabetic rat; D + IQ: diabetic rat treated with 1,5-isoquinolinediol. ${ }^{*} P<0.05$ compared with nondiabetic rat. ${ }^{\#} P<0.05$ compared with diabetic rat.

and GS is ROS, which is produced by high glucose in the retina [36]. It has been proposed that diabetic-related retinal neuropathy is a result of impaired BDNF, synaptophysin, and GS expressions [3, 18, 26]. Previous studies demonstrated that one month of diabetes decreases the retinal expression of BDNF and synaptophysin $[1,3]$, and that the antioxidant lutein prevented BDNF and synaptophysin reduction and avoided increase in cleaved caspase- 3 in the diabetic retina [3] suggesting that local oxidative stress has a neurodegenerative influence in diabetic retina. In addition, previous studies indicated that the expression of GS was significantly decreased in the diabetic rat retinas $[17,18]$. Downregulation of GS activity resulted in elevated glutamate levels $[17,19,20]$, which might induce retinal neuropathy via glutamate excitotoxicity. We found that PARP inhibition by 1,5-isoquinolinediol significantly prevented the diabetesinduced downregulation in BDNF, synaptophysin, and GS and upregulation of cleaved caspase-3 expressions. The mechanism by which the PARP inhibitor prevents diabetesinduced changes in the expression of BDNF, synaptophysin, GS, and caspase- 3 in the retinas remains unclear. However, our findings suggested that inhibition of PARP that attenuates diabetes-induced changes in the expression of BDNF, synaptophysin, GS, and caspase- 3 could be mediated by attenuating ROS generation. 


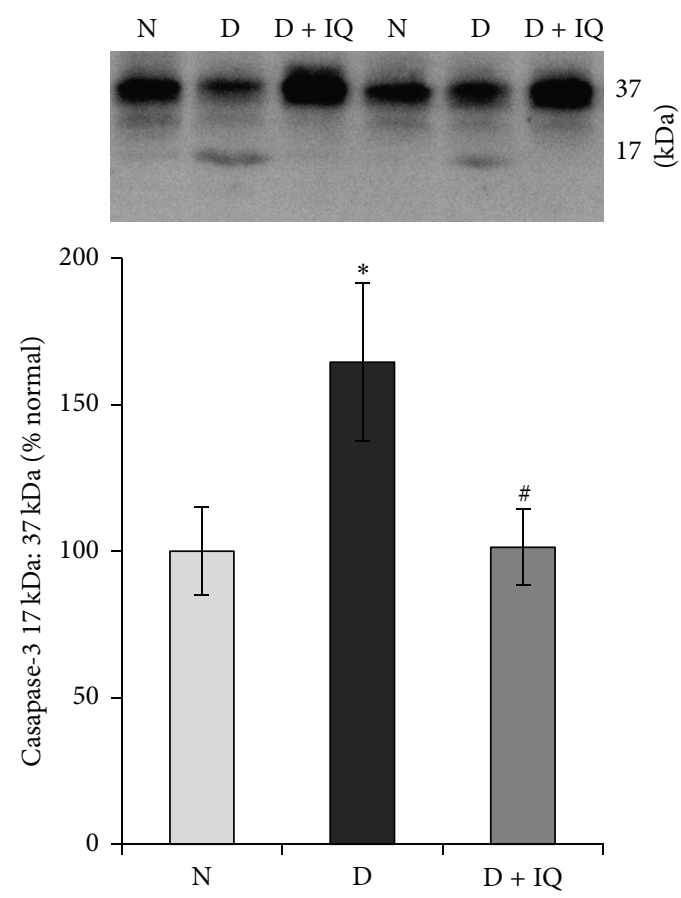

FIGURE 6: Effect of 1,5-isoquinolinediol on retinal cleavage of caspase-3 in diabetes. Cleavage of caspase-3 was measured by Western blot analysis, and the ratio of active caspase- $3(17 \mathrm{KDa})$ and pro-caspase-3 $(37 \mathrm{kDa})$ was calculated in control and diabetic rats maintained with and without PARP inhibitor treatment. Measurements were made in duplicate in six to eight rats in each group. Western blots are representative of three different experiments. Results are expressed as mean \pm SD. Values obtained from nondiabetic rats are considered as $100 \%$. N: nondiabetic rat; D: diabetic rat; D + IQ: diabetic rat treated with 1,5 -isoquinolinediol. ${ }^{*} P<0.05$ compared with nondiabetic rat. ${ }^{\#} P<0.05$ compared with diabetic rat.

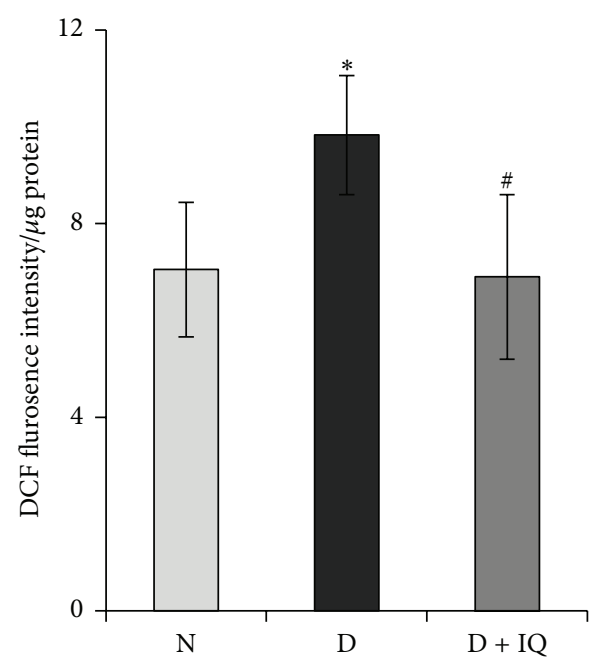

FIGURE 7: Effect of 1,5-isoquinolinediol on retinal reactive oxygen species (ROS) levels in diabetes. Freshly prepared retinal homogenates were incubated with DCHFDA $(5 \mu \mathrm{M})$ for $30 \mathrm{~min}$. An equal amount of protein was used to quantitate $2^{\prime}, 7^{\prime}$-dichlorofluorescein fluorescence. $\mathrm{N}$ : nondiabetic rat; D: diabetic rat; D + IQ: diabetic rat treated with 1,5-isoquinolinediol. Data are expressed as percent control and mean \pm SD from retina from 5-6 rats in each group. ${ }^{*} P<0.05$ compared with nondiabetic rat. ${ }^{\#} P<0.05$ compared with diabetic rat.

An additional mechanism that may contribute to the protective effect of PARP inhibition could be related to the suppression of $\mathrm{ERK}_{1 / 2}$ activation. $\mathrm{ERK}_{1 / 2}$ is a protein kinase intracellular signaling molecule that regulates the expression of genes involved in cell survival, apoptosis, and inflammatory response. Inhibition of ERK activation in the retina by its specific inhibitor U0126 blocks the actions of diabetes that causes upregulation of VEGF and inflammatory biomarkers, suggesting that ERK activation is involved in the pathogenesis of diabetic retinal dysfunction $[37,38]$. Moreover, a previous study also documented that inhibition of ERK activation in the retina by lutein blocks the actions 

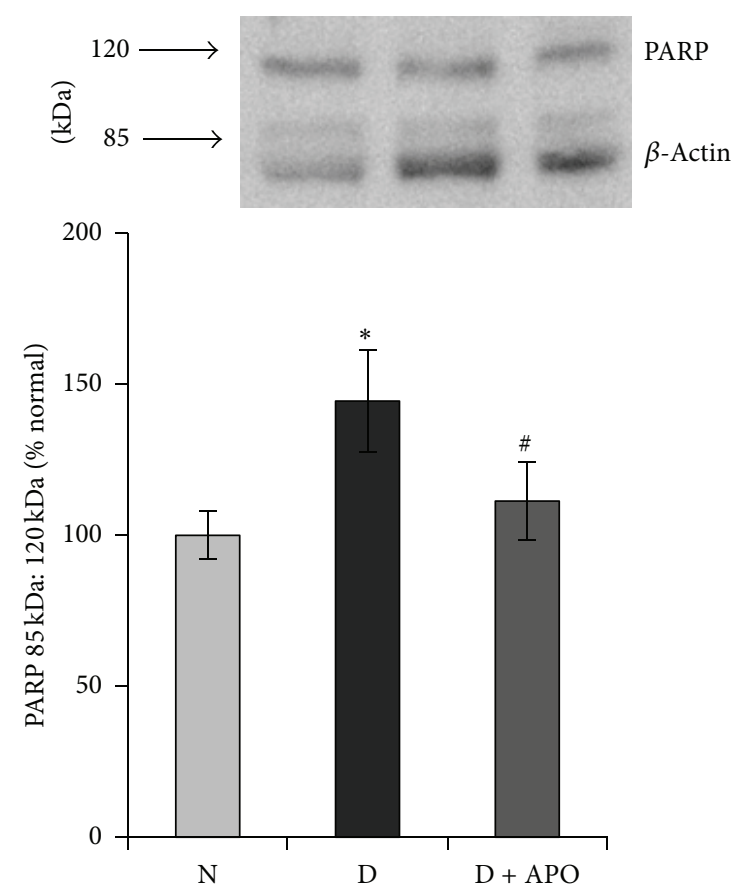

FIGURE 8: Diabetes-induced retinal PARP-1/2 activation were prevented by apocynin. PARP-1/2 expression in retina was quantified by Western blotting analysis using $\beta$-actin as a loading protein. Each measurement was made in duplicate or triplicate. The values are represented as mean \pm SD of 5-7 rats in each of the three groups. $\mathrm{N}$ : normal, D: Diabetes, D + Apo: Diabetic rat received apocynin. ${ }^{*} P<0.05$ compared to normal and ${ }^{\#} P<0.05$ compared to diabetes.

of diabetes that cause BDNF depletion and synaptophysin reduction [3]. It is known that the PARP activity is mediated by ERK-dependent phosphorylation [39-41] and persistent ERK activation has been shown in many complication-prone tissues in diabetes, including vascular smooth muscle cells, endothelial cells, peripheral nerves, and pericytes [42-44]. Here, we report that $\mathrm{ERK}_{1 / 2}$ is activated in the retinas of diabetic rats and 1,5-isoquinolinediol treatment inhibits ERK $_{1 / 2}$ activation, thus suggesting that ERK activation may be a target of diabetes-associated PARP activation. Consistent with our findings, previous studies demonstrated that PARP1 inhibition prevents ERK $_{1 / 2}$ phosphorylation $[45,46]$, but the signaling events downstream of PARP-1 activation are not fully identified. The mechanism by which the PARP inhibitor prevents diabetes-induced $\mathrm{ERK}_{1 / 2}$ phosphorylation in the retinas could be by lowering ROS generation as $\mathrm{ERK}_{1 / 2}$ activation is known to be induced by oxidative stress [47-49].

In conclusion, these data suggest that PARP activation mediates diabetes-induced increased oxidative stress, downregulation of BDNF, synaptophysin, and GS, and upregulation of caspase-3. Collectively, our present data suggest that blocking PARP signaling pathways might be a novel therapeutic strategy for neuronal dysfunction in visionthreatening diabetic retinopathy.

\section{Conflict of Interests}

The authors declare that they have no conflict of interests.

\section{Acknowledgments}

The authors thank Ms. Connie B. Unisa-Marfil for secretarial work. This work was supported by Dr. Nasser Al-Rashid, Research Chair in Ophthalmology (A. M. Abu El-Asrar).

\section{References}

[1] M. Seki, T. Tanaka, H. Nawa et al., "Involvement of brainderived neurotrophic factor in early retinal neuropathy of streptozotocin-induced diabetes in rats: therapeutic potential of brain-derived neurotrophic factor for dopaminergic amacrine cells," Diabetes, vol. 53, no. 9, pp. 2412-2419, 2004.

[2] A. J. Barber, "A new view of diabetic retinopathy: a neurodegenerative disease of the eye," Progress in NeuroPsychopharmacology and Biological Psychiatry, vol. 27, no. 2, pp. 283-290, 2003.

[3] M. Sasaki, Y. Ozawa, T. Kurihara et al., "Neurodegenerative influence of oxidative stress in the retina of a murine model of diabetes," Diabetologia, vol. 53, no. 5, pp. 971-979, 2010.

[4] R. Simó, C. Hernández, and European consortium for the early treatment of diabetic retinopathy (EUROCONDOR), "Neurodegeneration is an early event in diabetic retinopathy: therapeutic implications," British Journal of Ophthalmology, vol. 96, no. 10, pp. 1285-1290, 2012.

[5] J. M. Santos, G. Mohammad, Q. Zhong, and R. A. Kowluru, "Diabetic retinopathy, superoxide damage and antioxidants," Current Pharmaceutical Biotechnology, vol. 12, no. 3, pp. 352361, 2011.

[6] R. A. Kowluru and M. Kanwar, "Effects of curcumin on retinal oxidative stress and inflammation in diabetes," Nutrition and Metabolism, vol. 4, article 8, 2007.

[7] R. A. Kowluru, B. Menon, and D. L. Gierhart, "Beneficial effect of zeaxanthin on retinal metabolic abnormalities in diabetic rats," Investigative Ophthalmology and Visual Science, vol. 49, no. 4, pp. 1645-1651, 2008.

[8] G. Mohammad and R. A. Kowluru, "Matrix metalloproteinase2 in the development of diabetic retinopathy and mitochondrial dysfunction," Laboratory Investigation, vol. 90, no. 9, pp. 1365$1372,2010$.

[9] H. Zong, M. Ward, and A. W. Stitt, "AGEs, RAGE, and diabetic retinopathy," Current Diabetes Reports, vol. 11, no. 4, pp. 244252, 2011.

[10] K. C. Silva, M. A. B. Rosales, S. K. Biswas, J. B. L. De Faria, and J. M. L. De Faria, "Diabetic retinal neurodegeneration is associated with mitochondrial oxidative stress and is improved by an angiotensin receptor blocker in a model combining hypertension and diabetes," Diabetes, vol. 58 , no. 6, pp. $1382-$ 1390, 2009.

[11] M. Seki, H. Nawa, T. Fukuchi, H. Abe, and N. Takei, "BDNF is upregulated by postnatal development and visual experience: quantitative and immunohistochemical analyses of BDNF in the rat retina," Investigative Ophthalmology and Visual Science, vol. 44, no. 7, pp. 3211-3218, 2003.

[12] K. R. G. Martin, H. A. Quigley, D. J. Zack et al., "Gene therapy with brain-derived neurotrophic factor as a protection: retinal ganglion cells in a rat glaucoma model," Investigative Ophthalmology and Visual Science, vol. 44, no. 10, pp. 43574365, 2003.

[13] D. K. Binder and H. E. Scharfman, "Brain-derived neurotrophic factor," Growth Factors, vol. 22, no. 3, pp. 123-131, 2004. 
[14] T. C. Nag and S. Wadhwa, "Differential expression of syntaxin-1 and synaptophysin in the developing and adult human retina," Journal of Biosciences, vol. 26, no. 2, pp. 179-191, 2001.

[15] T. Kivela, A. Tarkkanen, and I. Virtanen, "Synaptophysin in the human retina and retinoblastoma. An immunohistochemical and Western blotting study," Investigative Ophthalmology and Visual Science, vol. 30, no. 2, pp. 212-219, 1989.

[16] S. C. Massey, "Cell types using glutamate as a neurotransmitter in the vertebrate retina," Progress in Retinal Research, vol. 9, pp. 399-425, 1990.

[17] E. Lieth, K. F. LaNoue, D. A. Antonetti, and M. Ratz, "Diabetes reduces glutamate oxidation and glutamine synthesis in the retina," Experimental Eye Research, vol. 70, no. 6, pp. 723-730, 2000.

[18] Y. Xu-hui, Z. Hong, W. Yu-hong, L. Li-juan, T. Yan, and L. Ping, "Time-dependent reduction of glutamine synthetase in retina of diabetic rats," Experimental Eye Research, vol. 89, no. 6, pp. 967-971, 2009.

[19] E. Lieth, A. J. Barber, B. Xu et al., "Glial reactivity and impaired glutamate metabolism in short- term experimental diabetic retinopathy," Diabetes, vol. 47, pp. 815-820, 1998.

[20] R. A. Kowluru, R. L. Engerman, G. L. Case, and T. S. Kern, "Retinal glutamate in diabetes and effect of antioxidants," Neurochemistry International, vol. 38, no. 5, pp. 385-390, 2001.

[21] Y. Ozawa, T. Kurihara, M. Sasaki et al., "Neural degeneration in the retina of the streptozotocin-induced type 1 diabetes model," Experimental Diabetes Research, vol. 2011, Article ID 108328, 2011.

[22] L. Zheng, C. Szabó, and T. S. Kern, "Poly(ADP-ribose) polymerase is involved in the development of diabetic retinopathy via regulation of nuclear factor- $\kappa \mathrm{B}$," Diabetes, vol. 53 , no. 11, pp. 2960-2967, 2004.

[23] R. Sugawara, T. Hikichi, N. Kitaya et al., "Peroxynitrite decomposition catalyst, FP15, and poly(ADP-ribose) polymerase inhibitor, PJ34, inhibit leukocyte entrapment in the retinal microcirculation of diabetic rats," Current Eye Research, vol. 29, no. 1, pp. 11-16, 2004.

[24] X. Qin, Z. Zhang, H. Xu, and Y. Wu, "Notch signaling protects retina from nuclear factor- $\kappa \mathrm{B}$ - and poly-ADP-ribosepolymerase-mediated apoptosis under high-glucose stimulation," Acta Biochimica et Biophysica Sinica, vol. 43, no. 9, pp. 703-711, 2011.

[25] I. G. Obrosova and U. A. Julius, "Role for poly(ADP-ribose) polymerase activation in diabetic nephropathy, neuropathy and retinopathy," Current Vascular Pharmacology, vol. 3, no. 3, pp. 267-283, 2005.

[26] I. G. Obrosova, V. R. Drel, P. Pacher et al., “Oxidative-nitrosative stress and poly(ADP-ribose) polymerase (PARP) activation in experimental diabetic neuropathy: the relation is revisited," Diabetes, vol. 54, no. 12, pp. 3435-3441, 2005.

[27] S. Lupachyk, H. Shevalye, Y. Maksimchyk, V. R. Drel, and I. G. Obrosova, "PARP inhibition alleviates diabetes-induced systemic oxidative stress and neural tissue 4-hydroxynonenal adduct accumulation: correlation with peripheral nerve function," Free Radical Biology and Medicine, vol. 50, no. 10, pp. 1400-1409, 2011.

[28] I. G. Obrosova, A. G. Minchenko, R. N. Frank et al., "Poly(ADP-ribose) polymerase inhibitors counteract diabetesand hypoxia-induced retinal vascular endothelial growth factor overexpression," International journal of molecular medicine, vol. 14, no. 1, pp. 55-64, 2004.
[29] B. Dasari, J. R. Prasanthi, G. Marwarha, B. B. Singh, and O. Ghribi, "Cholesterol-enriched diet causes age-related macular degeneration-like pathology in rabbit retina," BMC Ophthalmology, vol. 11, no. 1, article 22, 2011.

[30] M. A. Cotter and N. E. Cameron, "Effect of the NAD $(\mathrm{P}) \mathrm{H}$ oxidase inhibitor, apocynin, on peripheral nerve perfusion and function in diabetic rats," Life Sciences, vol. 73, no. 14, pp. 18131824, 2003.

[31] P. Jagtap and C. Szabo, "Poly(ADP-ribose) polymerase and the therapeutic effects of its inhibitors," Nature Reviews Drug Discovery, vol. 4, no. 5, pp. 421-440, 2005.

[32] I. G. Obrosova, V. R. Drel, A. K. Kumagai, C. Szábo, P. Pacher, and M. J. Stevens, "Early diabetes-induced biochemical changes in the retina: comparison of rat and mouse models," Diabetologia, vol. 49, no. 10, pp. 2525-2533, 2006.

[33] Z. Zheng, H. Chen, H. Wang et al., "Improvement of retinal vascular injury in diabetic rats by statins is associated with the inhibition of mitochondrial reactive oxygen species pathway mediated by peroxisome proliferator-activated receptor $\gamma$ coactivator 1 $\alpha$," Diabetes, vol. 59, no. 9, pp. 2315-2325, 2010.

[34] Z. H. Wan, W. Z. Li, Y. Z. Li et al., "Poly(ADP-Ribose) polymerase inhibition improves erectile function in diabetic rats," Journal of Sexual Medicine, vol. 8, no. 4, pp. 1002-1014, 2011.

[35] C. Szabó, A. Biser, R. Benko, E. Böttinger, and K. Suszták, "Poly(ADP-ribose) polymerase inhibitors ameliorate nephropathy of type 2 diabetic Leprdb/db mice," Diabetes, vol. 55, no. 11, pp. 3004-3012, 2006.

[36] M. S. Ola, M. I. Nawaz, H. A. Khan, and A. S. Alhomida, "Neurodegeneration and neuroprotection in diabetic retinopathy," International Journal of Molecular Sciences, vol. 14, no. 2, pp. 2559-2572, 2013.

[37] X. Ye, G. Xu, Q. Chang et al., "ERK1/2 signaling pathways involved in VEGF release in diabetic rat retina," Investigative Ophthalmology and Visual Science, vol. 51, no. 10, pp. 5226-5233, 2010.

[38] G. Mohammad, M. Siddiquei, M. Imtiaz Nawaz, and A. M. Abu El-Asrar, "The ERK1/2 inhibitor U0126 attenuates diabetesinduced upregulation of MMP-9 and biomarkers of inflammation in the retina," Journal of Diabetes Research, vol. 2013, Article ID 658548, 9 pages, 2013.

[39] M. Domercq, S. Mato, F. N. Soria, M. V. Sánchez-gómez, E. Alberdi, and C. Matute, "Zn2+ -induced ERK activation mediates PARP-1-dependent ischemic-reoxygenation damage to oligodendrocytes," Glia, vol. 61, no. 3, pp. 383-393, 2013.

[40] T. T. T. Nguyen, E. Tran, T. H. Nguyen, P. T. Do, T. H. Huynh, and H. Huynh, "The role of activated MEK-ERK pathway in quercetin-induced growth inhibition and apoptosis in A549 lung cancer cells," Carcinogenesis, vol. 25, no. 5, pp. 647-659, 2004 .

[41] J. Liu, W. Mao, B. Ding, and C.-S. Liang, "ERKs/p53 signal transduction pathway is involved in doxorubicin-induced apoptosis in H9c2 cells and cardiomyocytes," The American Journal of Physiology: Heart and Circulatory Physiology, vol. 295, no. 5, pp. H1956-H1965, 2008.

[42] J. Yang, Y. Han, H. Sun et al., “(-)-Epigallocatechin gallate suppresses proliferation of vascular smooth muscle cells induced by high glucose by inhibition of PKC and ERK1/2 signalings," Journal of Agricultural and Food Chemistry, vol. 59, no. 21, pp. 11483-11490, 2011. 
[43] L. Stenberg, M. Kanje, L. Mårtensson, and L. B. Dahlin, "Injuryinduced activation of ERK $1 / 2$ in the sciatic nerve of healthy and diabetic rats," NeuroReport, vol. 22, no. 2, pp. 73-77, 2011.

[44] G. Mohammad and R. A. Kowluru, "Diabetic retinopathy and signaling mechanism for activation of matrix metalloproteinase-9," Journal of Cellular Physiology, vol. 227, no. 3, pp. 1052-1061, 2012.

[45] B. Veres, B. Radnai, F. Gallyas Jr. et al., "Regulation of kinase cascades and transcription factors by a poly(ADPribose) polymerase-1 inhibitor, 4-hydroxyquinazoline, in lipopolysaccharide-induced inflammation in mice," Journal of Pharmacology and Experimental Therapeutics, vol. 310, no. 1, pp. 247-255, 2004.

[46] C. Éthier, Y. Labelle, and G. G. Poirier, "PARP-1-induced cell death through inhibition of the MEK/ERK pathway in MNNGtreated HeLa cells," Apoptosis, vol. 12, no. 11, pp. 2037-2049, 2007.

[47] Y. Luo and D. B. DeFranco, "Opposing roles for ERK1/2 in neuronal oxidative toxicity: distinct mechanisms of ERK1/2 action at early versus late phases of oxidative stress," Journal of Biological Chemistry, vol. 281, no. 24, pp. 16436-16442, 2006.

[48] T. P. Rygiel, A. E. Mertens, K. Strumane, R. van der Kammen, and J. G. Collard, "The Rac activator Tiam1 prevents keratinocyte apoptosis by controlling ROS-mediated ERK phosphorylation," Journal of Cell Science, vol. 121, no. 8, pp. 1183-1192, 2008.

[49] X. Wang, Z. Wang, Y. Yao et al., "Essential role of ERK activation in neurite outgrowth induced by $\alpha$-lipoic acid," Biochimica et Biophysica Acta, vol. 1813, no. 5, pp. 827-838, 2011. 


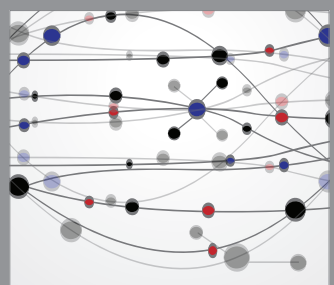

The Scientific World Journal
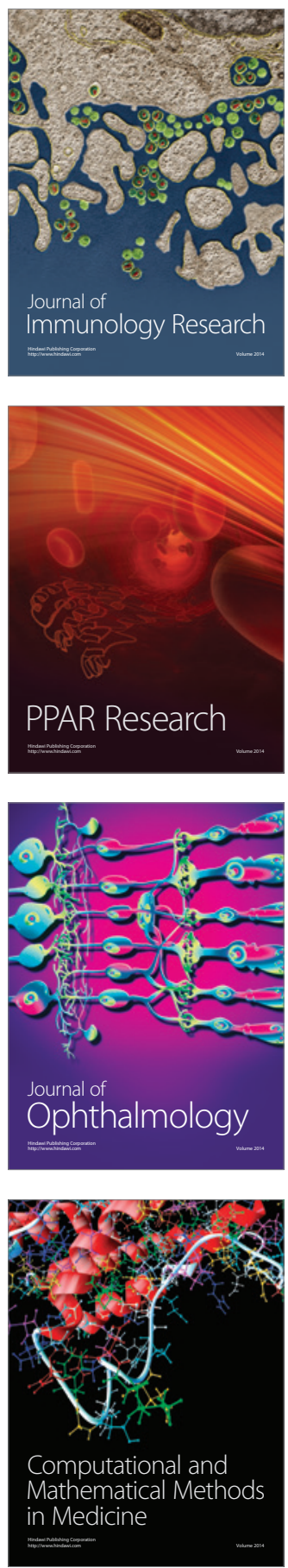

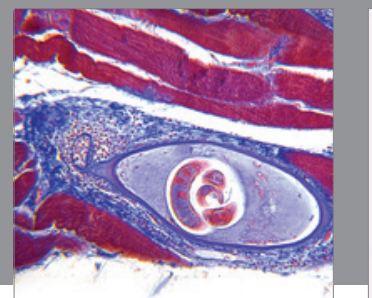

Gastroenterology

Research and Practice
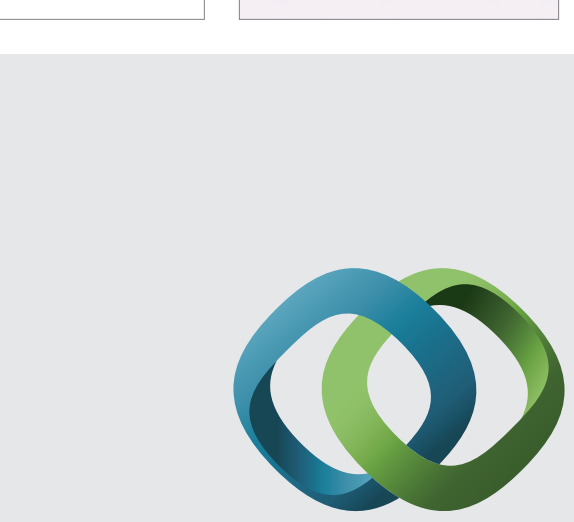

\section{Hindawi}

Submit your manuscripts at

http://www.hindawi.com
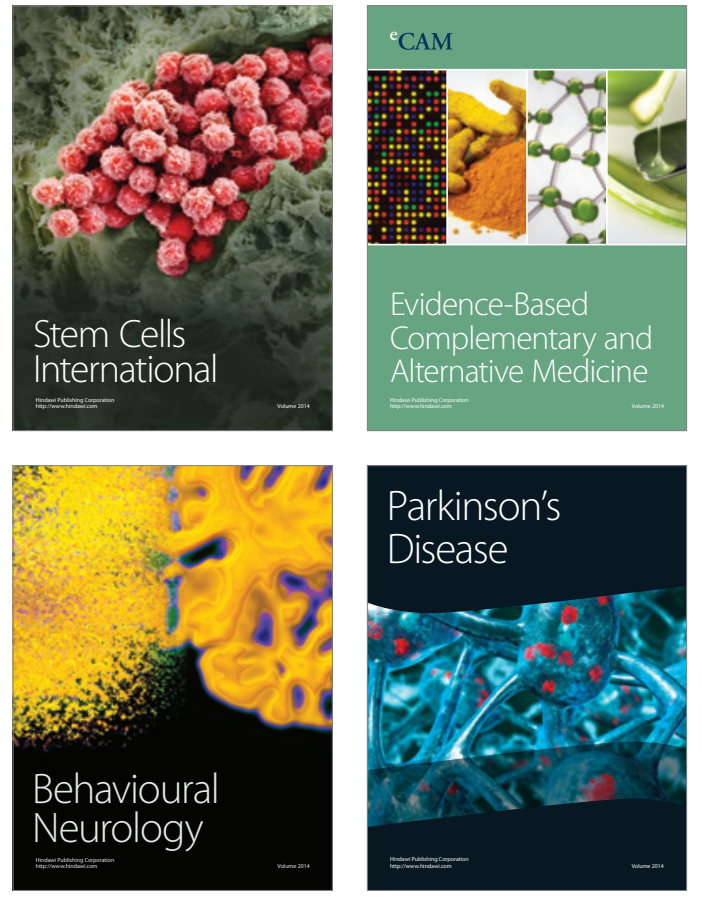
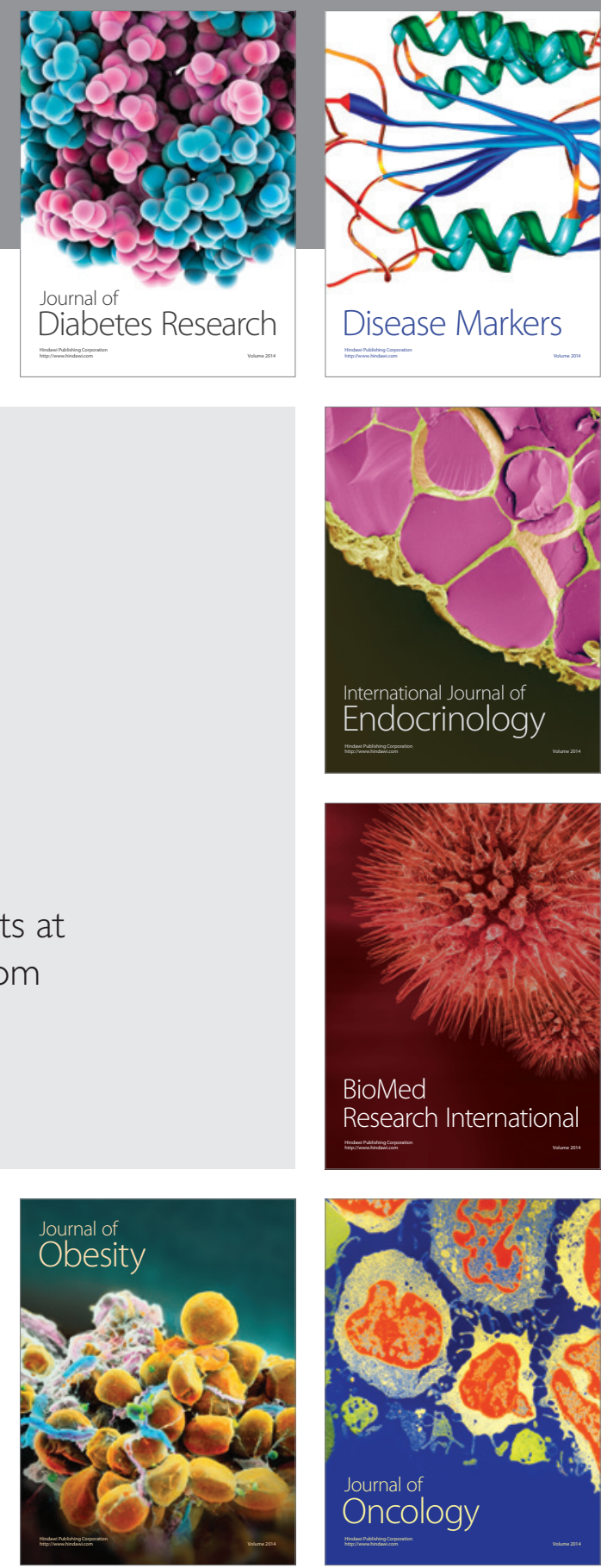

Disease Markers
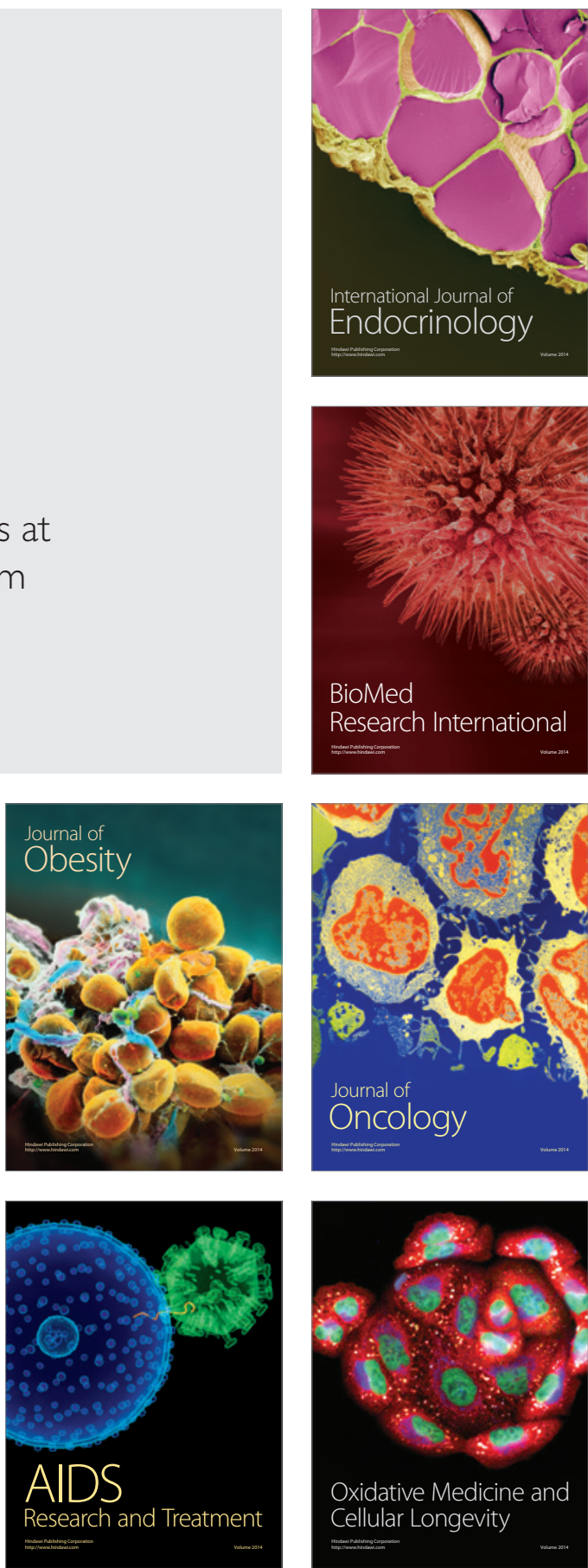